\title{
DIFFERENTIAL RECURSION RELATIONS FOR LAGUERRE FUNCTIONS ON HERMITIAN MATRICES
}

\author{
MARK DAVIDSON AND GESTUR ÓLAFSSON
}

\begin{abstract}
In our previous papers [1] 2] we studied Laguerre functions and polynomials on symmetric cones $\Omega=H / L$. The Laguerre functions $\ell_{\mathbf{n}}^{\nu}, \mathbf{n} \in \boldsymbol{\Lambda}$, form an orthogonal basis in $L^{2}\left(\Omega, d \mu_{\nu}\right)^{L}$ and are related via the Laplace transform to an orthogonal set in the representation space of a highest weight representations $\left(\pi_{\nu}, \mathcal{H}_{\nu}\right)$ of the automorphism group $G$ corresponding to a tube domain $T(\Omega)$. In this article we consider the case where $\Omega$ is the space of positive definite Hermitian matrices and $G=\mathrm{SU}(n, n)$. We describe the Lie algebraic realization of $\pi_{\nu}$ acting in $L^{2}\left(\Omega, d \mu_{\nu}\right)$ and use that to determine explicit differential equations and recurrence relations for the Laguerre functions.
\end{abstract}

\section{INTRODUCTION}

In this article we continue our study of Laguerre functions and Laguerre polynomials on symmetric cones. Let $\nu>0$ and $\alpha=\nu-1$. It is an old and well known fact that the classical Laguerre functions $\ell_{n}^{\alpha}(t)=e^{-t} L_{n}^{\alpha}(2 t)$ form an orthogonal basis for $L^{2}\left(\mathbb{R}^{+}, t^{\alpha} d t\right)$ and satisfy several differential equations and recursion relations. The same holds for the Laguerre polynomials $L_{n}^{\alpha}(t)$, in fact they may be defined as the polynomial solution to a second order differential operator known as Laguerre's differential operator. In our previous article [1] it was shown that the representation theory of $G=\widetilde{\mathrm{SL}(2, \mathbb{R})}$, the universal covering group of $\mathrm{SL}(2, \mathbb{R})$, unified many of the disparate properties of Laguerre functions. Briefly, the Laguerre functions $\ell_{n}^{\alpha}$ form a basis of the space of $K=\widetilde{\mathrm{SO}(2)}$-finite vectors of a highest weight representations $T_{\alpha}$ of $G$ acting on $L^{2}\left(\mathbb{R}^{+}, t^{\alpha} d t\right)$. The Lie algebra $\mathfrak{s l}(2, \mathbb{C})$ acts by at most second order differential operators on the space of smooth vectors of those representations. In particular, the elements

$$
X^{-}=\left(\begin{array}{cc}
1 & i \\
i & -1
\end{array}\right), \quad X^{+}=\left(\begin{array}{cc}
-1 & i \\
i & 1
\end{array}\right)=-\overline{X^{-}}, \quad \text { and } \quad X^{0}=\left(\begin{array}{cc}
0 & -i \\
i & 0
\end{array}\right)
$$

act, respectively, by the differential operators

$$
\begin{aligned}
D^{-} & =t D^{2}+(2 t+\nu) D+(t+\nu) \\
D^{+} & =t D^{2}-(2 t-\nu) D+(t-\nu) \\
D^{0} & =t D^{2}+\nu D-t .
\end{aligned}
$$

Notice that $X^{0}$ is a basis for $\mathfrak{s o}(2)$ and that our normalization is such that

$$
\left[X^{0}, X^{-}\right]=2 X^{-}, \quad\left[X^{0}, X^{+}\right]=-2 X^{+}, \quad \text { and } \quad\left[X^{+}, X^{-}\right]=4 X^{0} .
$$

Key words and phrases. Holomorphic discrete series, highest weight representations, symmetric cones, orthogonal polynomials, Laguerre functions and polynomials, Laplace transform.

Research by G. Ólafsson supported by NSF grant DMS-0070607 and DMS-0139783. 
Furthermore, the action of $D^{0}$ gives us the following differential equation for the Laguerre functions:

$$
\left(t D^{2}+\nu D-t\right) \ell_{n}^{\alpha}=-(2 n+\nu) \ell_{n}^{\alpha}
$$

and the actions of the annihilating operator $D^{-}$and the creation operator $D^{+}$give us the recurrence relations for the Laguerre functions:

$$
D^{-} \ell_{n}^{\alpha}=\left(t D^{2}+(2 t+\nu) D+(t+\nu)\right) \ell_{n}^{\alpha}=-2(n+\nu-1) \ell_{n-1}^{\alpha}
$$

and

$$
D^{+} \ell_{n}^{\alpha}=\left(t D^{2}-(2 t-\nu) D+(t-\nu)\right) \ell_{n}^{\alpha}=-2(n+1) \ell_{n+1}^{\alpha} .
$$

These equations are transparent in the natural setting where the group acts on holomorphic functions on the upper half plane $\mathbb{C}^{+}=\{z=x+i y \mid y>0\}$. That natural setting is related to $L^{2}\left(\mathbb{R}^{+}, t^{\alpha} d t\right)$ by the Laplace transform $\mathcal{L}_{\nu}(f)(z)=$ $\int_{\mathbb{R}^{+}} e^{-z t} f(t) t^{\alpha} d t$ which we derived by a simple application of the restriction principle (see [1, 2, 7]). The Laplace transform is then used to transfer results from the holomorphic setting to $L^{2}\left(\mathbb{R}^{+}, t^{\alpha} d t\right)$. Notice that the automorphism group of the cone $\mathbb{R}^{+}$is in this case

$$
H \simeq\left\{\left(\begin{array}{cc}
e^{t} & 0 \\
0 & e^{-t}
\end{array}\right)=\exp (t Z) \mid t \in \mathbb{R}\right\}
$$

where

$$
Z=\left(\begin{array}{cc}
1 & 0 \\
0 & -1
\end{array}\right)=\frac{1}{2}\left(X^{-}-X^{+}\right) .
$$

The corresponding differential operator is $2 t D+\nu$ and we get

$$
(2 t D+\nu) \ell_{n}^{\alpha}=(n+1) \ell_{n+1}^{\alpha}-(n+\nu-1) \ell_{n-1}^{\alpha}
$$

which is, up to normalization of the Laguerre functions, the recursion relation stated in general in Theorem 7.9 and Remark 7.10 in [2], see equation (8) below.

In our article 2] we explore, among other things, a generalization and extension of these results to the setting where $\mathbb{R}^{+}$is replaced by a symmetric cone $H / L=$ $\Omega$, contained in a Euclidean Jordan algebra $J$, and $\operatorname{SL}(2, \mathbb{R})$ is replaced by the Hermitian group $G=\operatorname{Aut}(T(\Omega))_{o}$, where $T(\Omega)=\Omega+i J$. Furthermore, the measure $t^{\alpha} d t$ is replaced by $d \mu_{\nu}(t)=\Delta(t)^{\nu-d / r} d t$, where $\Delta$ is the determinant function of $J, d=\operatorname{dim}(J)$, and $r$ is the rank of $J$ (see 3 for the structure theory of Jordan algebras). In this setting the Laguerre functions form an orthogonal basis for the space of $L$-invariant functions $L^{2}\left(\Omega, d \mu_{\nu}\right)^{L}$. And again, the differential operators and recurrence relations that they satisfy can be derived from the action of the complexified Lie algebra $\mathfrak{g}_{\mathbb{C}}$ of $G$. If $\mathfrak{g}_{\mathbb{C}}^{L}$ denotes the $L$-invariant elements of $\mathfrak{g}_{\mathbb{C}}$ then $\mathfrak{g}_{\mathbb{C}}^{L}$ acts on the space of Laguerre functions. If $\mathfrak{g}$ is simple then $\operatorname{dim}\left(\mathfrak{g}_{\mathbb{C}}^{L}\right)=3$ and in fact there is a Lie algebra isomorphism

$$
\varphi: \mathfrak{g}_{\mathbb{C}}^{L} \rightarrow \mathfrak{s l}(2, \mathbb{C}) .
$$

We use the same notation as before and let $X^{0}$ be taken from the center of $\mathfrak{k}$, where $\mathfrak{k}$ is the Lie algebra of a maximal compact subgroup $K$ of $G$, containing $L$. If $\sigma: \mathfrak{g}_{\mathbb{C}} \rightarrow \mathfrak{g}_{\mathbb{C}}$ is the conjugation with respect to the real form $\mathfrak{g}$ and $\theta$ is the Cartan involution such that $G^{\theta}=K$, then we can choose the isomorphism $\varphi$ such that $\varphi(\sigma(X))=\overline{\varphi(X)}$ and $\varphi(\theta(X))=-\varphi(X)^{t}$. We then fix $X^{0}$ such that

$$
\varphi\left(X^{0}\right)=\left(\begin{array}{cc}
0 & -i \\
i & 0
\end{array}\right) .
$$


The operator $\operatorname{ad}\left(X^{0}\right): \mathfrak{g}_{\mathbb{C}} \rightarrow \mathfrak{g}_{\mathbb{C}}$ has eigenvalues 0,2 , and -2 . The eigenspace corresponding to the eigenvalue 0 is $\mathfrak{k}_{\mathbb{C}}$ whereas the eigenspace $\mathfrak{p}^{ \pm}$corresponding to the eigenvalue \pm 2 is an abelian Lie algebra and $\sigma\left(\mathfrak{p}^{+}\right)=\mathfrak{p}^{-}$. In particular, $\mathfrak{p}^{+} \cap \mathfrak{p}^{-}=\{0\}$. We choose $X^{\mp} \in\left(\mathfrak{p}^{ \pm}\right)^{L}$ such that

$$
\varphi\left(X^{-}\right)=\left(\begin{array}{cc}
1 & i \\
i & -1
\end{array}\right), \text { and } \varphi\left(X^{+}\right)=\left(\begin{array}{cc}
-1 & i \\
i & 1
\end{array}\right) .
$$

Then $Z=\frac{1}{2}\left(X^{-}-X^{+}\right)$is in the center of $\mathfrak{h}$. In [2] it was shown, that $Z$ corresponds to the differential operator

$$
E_{\nu}=\nu r+2 E
$$

where $E$ is the Euler operator $E f(x)=\left.\frac{d}{d t} f(t x)\right|_{t=1}$. Furthermore, the following difference equation was derived:

$$
2 E_{\nu} \ell_{\mathbf{m}}^{\nu}=\sum_{j=1}^{r} c_{\mathbf{m}}(j) \ell_{\mathbf{m}+\gamma_{j}}^{\nu}-\sum_{j=1}^{r}\left(\begin{array}{c}
\mathbf{m} \\
\mathbf{m}-\gamma_{j}
\end{array}\right)\left(\mathbf{m}_{j}-1+\nu-(j-1) \frac{d}{2}\right) \ell_{\mathbf{m}-\gamma_{j}}^{\nu}
$$

where $c_{m}(j)$ are certain constants. We notice that this equation includes the annihilation operator and the creation operator. It is one of our aims in this paper to separate the action of the creation and annihilation operators. We therefore return to the paradigm discussed earlier for $\operatorname{SL}(2, \mathbb{R})$ and carry out our extension for the group $\mathrm{SU}(n, n)$ acting on the tube type domain $T(\Omega)$, where $\Omega$ is the cone of positive definite $n \times n$ Hermitian matrices and $T(\Omega)$ is $\Omega+i \operatorname{Herm}(n)$. Using the Laplace transform we determine the action of $\mathfrak{g}_{\mathbb{C}}$ on $L^{2}\left(\Omega, d \mu_{\nu}\right)$. This calculation is carried out on $\mathfrak{k}_{\mathbb{C}}$, where $\mathfrak{k}$ is the Lie algebra of $K$, on $\mathfrak{p}^{+}$, and on $\mathfrak{p}^{-}$. The result is:

Theorem 4.1 Let $f \in L^{2}\left(\Omega, d \mu_{\nu}\right)$ be a smooth vector.

(1) Let $\left(\begin{array}{ll}a & b \\ b & a\end{array}\right) \in \mathfrak{k}_{\mathbb{C}}$. Then

$$
\lambda_{\nu}\left(\begin{array}{ll}
a & b \\
b & a
\end{array}\right) f(s)=\operatorname{tr}(-s \nabla b \nabla+(s a-a s-\nu b) \nabla+b s) f(s)
$$

(2) Let $\left(\begin{array}{cc}x & x \\ -x & -x\end{array}\right) \in \mathfrak{p}^{+}$. Then

$$
\lambda_{\nu}\left(\begin{array}{cc}
x & x \\
-x & -x
\end{array}\right) f(s)=\operatorname{tr}(s \nabla x \nabla+(\nu x+s x+x s) \nabla+(\nu x+s x)) f(s) .
$$

(3) Let $\left(\begin{array}{ll}x & -x \\ x & -x\end{array}\right) \in \mathfrak{p}^{-}$. Then

$$
\lambda_{\nu}\left(\begin{array}{cc}
x & -x \\
x & -x
\end{array}\right) f(s)=\operatorname{tr}(-s \nabla x \nabla+(-\nu x+s x+x s) \nabla+(\nu x-s x)) f(s) .
$$

Specializing to $\mathfrak{g}_{\mathbb{C}}^{L}=\operatorname{span}\left(X_{\circ}, X^{-}, X^{+}\right)$gives the analogue to Laguerres differential operator, the annihilation operator, and the creation operator. Specifically, we get

Theorem 5.1 The Laguerre functions are related by the following recursion relations:

(1) $\operatorname{tr}(-s \nabla \nabla-\nu \nabla+s) \ell_{\mathbf{m}}^{\nu}=(r \nu+|\mathbf{m}|) \ell_{\mathbf{m}}^{\nu}$. 
(2) $\frac{1}{2} \operatorname{tr}(s \nabla \nabla+(\nu I+2 s) \nabla+(\nu I+s)) \ell_{\mathbf{m}}^{\nu}(s)=-\sum_{j=1}^{r}\left(\begin{array}{c}\mathbf{m} \\ \mathbf{m}-\gamma_{\mathbf{j}}\end{array}\right)\left(m_{j}-1+\nu-\right.$ $\left.(j-1) \frac{d}{2}\right) \ell_{\mathbf{m}-\gamma_{\mathbf{j}}}^{\nu}$

(3) $\frac{1}{2} \operatorname{tr}(-s \nabla \nabla+(-\nu I+2 s) \nabla+(\nu I-s)) \ell_{\mathbf{m}}^{\nu}=\sum_{j=1}^{r} c_{\mathbf{m}}(j) \ell_{\mathbf{m}+\gamma_{\mathbf{j}}}^{\nu}$.

Several aspects of these formulas suggest an interpretation involving the structure theory of Jordan algebras. We have not pursued that here.

Our realization of the highest weight representations $\lambda_{\nu}$ in $L^{2}\left(\Omega, d \mu_{\nu}\right)$ has an interesting connection to the set of Whittaker vectors of the representation $\lambda_{\nu}$, see 6] 11. Recall that $\mathfrak{z}(\mathfrak{h})=\mathbb{R} Z$. Let

$$
\mathfrak{n}=\{X \in \mathfrak{g} \mid[Z, X]=2 X\} .
$$

Then $\mathfrak{n}$ is an abelian Lie algebra, and $\mathfrak{h} \oplus \mathfrak{n}=: \mathfrak{p}_{\max }$ is a maximal parabolic subalgebra in $\mathfrak{g}$. Let $P=\tilde{H} N$ be the corresponding maximal parabolic subgroup and notice that $\tilde{H}_{o}=H$. Let us recall here the construction of the isomorphism $\mathcal{H}_{\nu}(\Omega) \simeq L^{2}\left(\Omega, d \mu_{\nu}\right)$ where $\mathcal{H}_{\nu}(\Omega) \subset \mathcal{O}(T(\Omega))$ is the usual Hilbert space realization of the highest weight representation $\pi_{\nu}$. Then the restriction map $R(f):=\left.f\right|_{\Omega}$ is injective and intertwines in an obvious way the $\tilde{H}$-action on both sides. Now it is a well known fact that the cone $\Omega$ has a natural realization in the vector space $\mathfrak{n} \simeq N$ and therefore this map also intertwines in a natural way the $N$-action on both sides. It then follows that $\left(\pi_{\nu}, \mathcal{H}_{\nu}\right)$ can be realized as $\left(\tilde{\lambda}_{\nu}, L^{2}(\Omega, d t)\right)$ where we think now of $L^{2}(\Omega, d t)$ as a subspace of $L^{2}(N)$. The isomorphism $L^{2}\left(\Omega, d \mu_{\nu}\right) \simeq L^{2}(\Omega, d t)$ is here given by

$$
f \mapsto \Delta^{(\nu-d / r) / 2} f
$$

In our example of $G=\operatorname{SL}(2, \mathbb{R})$ we have $Z=\left(\begin{array}{cc}1 & 0 \\ 0 & -1\end{array}\right)$,

$$
N=\left\{\left(\begin{array}{ll}
1 & x \\
0 & 1
\end{array}\right) \mid x \in \mathbb{R}\right\} \simeq \mathbb{R} \text {. }
$$

and

$$
P= \pm\left\{\left(\begin{array}{cc}
a & x \\
0 & a^{-1}
\end{array}\right) \mid a>0, x \in \mathbb{R}\right\} .
$$

Furthermore, the isomorphism $L^{2}\left(\mathbb{R}^{+}, t^{\alpha} d t\right) \simeq L^{2}\left(\mathbb{R}^{+}, d t\right)$ is simply

$$
f \mapsto\left(t \mapsto t^{\alpha / 2} f(t)\right)
$$

Let

$$
X_{N}=\left(\begin{array}{ll}
0 & 1 \\
0 & 0
\end{array}\right)=\frac{-i}{2}\left(\frac{1}{2}\left(X^{+}+X^{-}\right)-X^{0}\right) .
$$

The set of equations (13) gives us then the following action of the Lie algebra on functions on $L^{2}\left(\mathbb{R}^{+}, d t\right)$ :

$$
\begin{aligned}
Z \cdot f(t) & =2 t f^{\prime}(t)+f(t) \\
X_{N} \cdot f(t) & =-t i f(t) \\
\left(i X_{0}\right) \cdot f(t) & =i\left(t f^{\prime \prime}(t)+f^{\prime}(t)-\left(\frac{\alpha^{2}}{4 t}+t\right) f(t)\right)
\end{aligned}
$$

These are exactly the equations in [6]. The motivation in [6] is different though. There, as well as in [1], the differential equations satisfied by the Laguerre functions were used to reconstruct the representation $\lambda_{\nu}$ whereas we have used the 
representation $\lambda_{\nu}$ to derive the differential equations that the Laguerre functions satisfy. It is now an interesting task to generalize this for the universal covering of the group $\mathrm{SU}(n, n)$. We remark the following. First notice that $r / d=1 / n$. The isomorphism $L^{2}\left(\Omega, \Delta(t)^{\nu-1 / n} d t\right) \simeq L^{2}(\Omega, d t)$ is again given by

$$
f \mapsto \Delta^{\frac{n \nu-1}{2 n}} f .
$$

Now one should use Theorem 4.1 and Proposition 3.2 which gives us the derivatives of $\Delta^{\frac{n \nu-1}{2 n}}$, to derive the the action of $\mathfrak{s u}(n, n)$ on $L^{2}(\Omega, d t)$.

After we submitted this article the paper of Ricci and Vignati [8] came to our attention. There a system of differential operators are defined that are diagonal on the Laguerre basis with different eigenvalues. This system generalizes the classical Laguerre operator, however, the raising and lowering operators are not discussed.

\section{1. $\mathrm{SU}(n, n)$ AND Its Lie Algebra}

We keep the notation from the introduction but specialize to the case $G=$ $\mathrm{SU}(n, n)$. The standard definition of $\mathrm{SU}(n, n)$ as found on page 444 of [5] acts naturally on the generalized unit disk. Our definition below is suited for the right half plane action and is conjugate to the standard version by the Cayley transform. The group $\mathrm{SU}(n, n)$ is thus defined as follows: Let $J=\left(\begin{array}{ll}0 & 1 \\ 1 & 0\end{array}\right)$. Then

$$
\mathrm{SU}(n, n)=\left\{g \in \mathrm{SL}(2 n, \mathbb{C}) \mid g J g^{*}=J\right\} .
$$

We frequently write $g \in S U(n, n)$ in block form as $g=\left(\begin{array}{ll}A & B \\ C & D\end{array}\right)$. We then have the following relations on the entries:

$$
\begin{aligned}
A B^{*}+B A^{*}=0 & & B^{*} D+D^{*} B=0 \\
C D^{*}+D C^{*}=0 & & A^{*} C+C^{*} A=0 \\
A D^{*}+B C^{*}=1 & & A^{*} D+C^{*} B=1 \\
D A^{*}+C B^{*}=1 & & B^{*} C+D^{*} A=1 .
\end{aligned}
$$

The cone $\Omega$ in $J=\operatorname{Herm}(n)$ can be identified as the set of positive definite Hermitian matrices. The group $\mathrm{SU}(n, n)$ acts on $T(\Omega)=\Omega+i J$ by linear fractional transformations:

$$
\left(\begin{array}{cc}
A & B \\
C & D
\end{array}\right) \cdot T=(A T+B)(C T+D)^{-1} .
$$

Let $\Gamma_{\Omega}(\nu)$ be the Gindikin-Koecher Gamma function associated with the cone $\Omega$, see [3] :

$$
\Gamma_{\Omega}(\nu)=\int_{\Omega} e^{-\operatorname{tr}(x)} \operatorname{det}(x)^{\nu-n} d x .
$$

Assume that $\nu>2 n-1$. Let $\mathcal{H}_{\nu}(T(\Omega))$ be the space of holomorphic functions $F: T(\Omega) \rightarrow \mathbb{C}$ such that

$$
\|F\|^{2}:=\alpha_{\nu} \int_{T(\Omega)}|F(x+i y)|^{2} \operatorname{det}(y)^{\nu-2 n} d x d y<\infty
$$

where

$$
\alpha_{\nu}=\frac{2^{n \nu}}{(4 \pi)^{n^{2}} \Gamma_{\Omega}(\nu-n)} .
$$


Then $\mathcal{H}_{\nu}(T(\Omega))$ is a Hilbert space and the group $\mathrm{SU}(n, n)$ acts unitarily and irreducibly on $\mathcal{H}_{\nu}(T(\Omega))$ by

$$
\pi_{\nu}(g) F(z)=J\left(g^{-1}, z\right)^{\frac{\nu}{2 n}} f\left(g^{-1} \cdot z\right),
$$

where $J\left(\left(\begin{array}{cc}A & B \\ C & D\end{array}\right), z\right)=\operatorname{det}(C z+D)^{-2 n}$, (see section 2.8 of [2] ). Let us also mention, that the Hilbert space structure and the unitary action extends analytically to the domain $\nu>n-1$, 9, 10.

The following table summarizes most aspects of the specialization from the introduction to $\mathrm{SU}(n, n)$, some of which we have already used:

\begin{tabular}{|c|c|}
\hline$G$ & $\mathrm{SU}(n, n)$ \\
\hline \hline$a$ & 2 \\
\hline$d$ & $n^{2}$ \\
\hline$r$ & $2 n$ \\
\hline$p$ & $\left(\begin{array}{cc}A & B \\
B & A\end{array}\right) \in \mathrm{SU}(n, n): A \pm B \in \mathrm{U}(n)$ \\
\hline$K$ & $\left(\begin{array}{c}A \\
0\end{array}\left(\begin{array}{c}\left.A^{*}\right)^{-1}\end{array}\right) \in \mathrm{SL}(2 n, \mathbb{C}): \quad A \in \mathrm{GL}(n, \mathbb{C})\right.$ \\
\hline$H$ & $\left(\begin{array}{cc}A & 0 \\
0 & A\end{array}\right) \in \mathrm{SL}(2 n, \mathbb{C}): \quad A \in \mathrm{SU}(n)$ \\
\hline$L$ & Herm(n): the $n \times n$ Hermitian matrices \\
\hline$\Omega$ & positive definite matrices in $J$ \\
\hline$\Delta$ & det \\
\hline
\end{tabular}

1.1. The Lie Algebra. The Lie algebra for $\mathrm{SU}(n, n)$ is

$$
\mathfrak{g}=\mathfrak{s u}(n, n)=\left\{\left(\begin{array}{ll}
a & b \\
c & d
\end{array}\right) \in \mathfrak{s l}(2 n, \mathbb{C}) \mid a=-d^{*}, b=-b^{*}, c=-c^{*}\right\} .
$$

Its complexification, $\mathfrak{g}_{\mathbb{C}}$, is the set $\mathfrak{s l}(2 n, \mathbb{C})$. Important Lie subalgebras are $\mathfrak{k}$, the Lie algebra of $K$, its complexification $\mathfrak{k}_{\mathbb{C}}$, and $\mathfrak{p}^{+}$and $\mathfrak{p}^{-}$, which act by annihilation and creation operators in $\mathcal{H}_{\nu}(T(\Omega))$. They are given as follows:

$$
\begin{aligned}
\mathfrak{k}=\left\{\left(\begin{array}{ll}
a & b \\
b & a
\end{array}\right) \mid a=-a^{*}, b=-b^{*}, \text { and } \operatorname{tr}(a)=0\right\}, \\
\mathfrak{k}_{\mathbb{C}}=\left\{\left(\begin{array}{cc}
a & b \\
b & a
\end{array}\right) \mid \operatorname{tr}(a)=0\right\}, \\
\mathfrak{p}^{+}=\left\{\left(\begin{array}{cc}
x & x \\
-x & -x
\end{array}\right) \mid x=x^{*}\right\},
\end{aligned}
$$


and

$$
\mathfrak{p}^{-}=\left\{\left(\begin{array}{cc}
x & -x \\
x & -x
\end{array}\right) \mid x=x^{*}\right\} .
$$

2. The actions of the subalgebras $\mathfrak{k}, \mathfrak{p}^{-}$, And $\mathfrak{p}^{+}$

In this section we give the action of the complexification, $\mathfrak{g}_{\mathbb{C}}$, of the Lie algebra of $G=\mathrm{SU}(n, n)$ on $\mathcal{H}_{\nu}(T(\Omega))$. The proof is a straightforward calculation and will not be given here. Recall though the basic definitions. Let $X \in \mathfrak{g}$ and let $F \in \mathcal{H}_{\nu}(T(\Omega))$ be a smooth vector. By definition

$$
\begin{aligned}
\pi_{\nu}(X) F(z) & =\left.\frac{d}{d t} \pi_{\nu}(\exp t X) F(z)\right|_{t=0} \\
& =\left.\frac{d}{d t} J(\exp -t X, z)^{\frac{\nu}{2 n}} F(\exp (-t X) \cdot z)\right|_{t=0} .
\end{aligned}
$$

The representation of $\mathfrak{g}_{\mathbb{C}}$ is defined by $\pi_{\nu}(X+i Y)=\pi_{\nu}(X)+i \pi_{\nu}(Y)$. But since functions in $\mathcal{H}_{\nu}(T(\Omega))$ are holomorphic we get that $\pi_{\nu}(X)$ is given by the same formula for $X \in \mathfrak{g}_{C}$.

Theorem 2.1. For $w \in V$ let $\delta(w)$ denote the non-normalized directional derivative in the direction $w$. Let $F$ be a $C^{\infty}$-vector in $\mathcal{H}_{\nu}(T(\Omega))$.

(1) Let $X=\left(\begin{array}{ll}a & b \\ b & a\end{array}\right) \in \mathfrak{k}_{\mathbb{C}}$. Then $\pi_{\nu}(X) F(z)=\nu \operatorname{tr}(b z) F(z)+\delta(z b z+z a-a z-b) F(z)$.

(2) Let $X=\left(\begin{array}{cc}x & x \\ -x & -x\end{array}\right) \in \mathfrak{p}^{+}$. Then

$$
\pi_{\nu}(X) F(z)=-\nu \operatorname{tr}(x(1+z)) F(z)-\delta((1+z) x(1+z)) F(z) .
$$

(3) Let $X=\left(\begin{array}{ll}x & -x \\ x & -x\end{array}\right) \in \mathfrak{p}^{-}$. Then

$$
\pi_{\nu}(X) F(z)=\nu \operatorname{tr}(x(z-1)) F(z)+\delta((z-1) x(z-1)) F(z) .
$$

\section{The GRADIEnT}

Our main results are expressed in terms of the gradient of complex valued function. This section establishes notation and well known results.

Let $V$ be a finite dimensional complex vector space with inner product $(\cdot \mid \cdot)$. Suppose $J$ is a real form on which the restriction of the inner product is a real inner product. Let $f: J \rightarrow \mathbb{R}$ be a differentiable function, i.e., all (nonnormalized) directional derivatives $D_{u}, u \in J$, exist. For $s \in J$, we define $\nabla f(s) \in J$ by the formula

$$
(\nabla f(s) \mid u)=D_{u} f(s) .
$$

For a $\mathbb{C}$-valued function $f=f_{1}+i f_{2}$ we define $\nabla f=\nabla f_{1}+i \nabla f_{2}$. For $z=x+i y \in V$, $x, y \in J$, we define $D_{z}=D_{x}+i D_{y}$. The following proposition is for the most part a consequence of these definitions.

Proposition 3.1. Let the notation be as above. Then the following holds:

(1) Let $f: J \rightarrow \mathbb{C}$ be differentiable. Then

$$
(\nabla f(s) \mid z)=D_{\bar{z}} f(s),
$$

where $\bar{z}$ is conjugation with respect to $J$. 
(2) If $f: J \rightarrow \mathbb{C}$ is the restriction of a $\mathbb{C}$-linear map $F: V \rightarrow \mathbb{C}$ to $J$, then $(\nabla f(s) \mid w)=F(\bar{w})$. In particular if $z, w \in V$ and $f(s)=(z \mid s)=(s \mid \bar{z})$ then $(\nabla f(s) \mid w)=([\nabla(z \mid s)] \mid w)=(z \mid w)$.

(3) If $f$ is the restriction of a holomorphic function on $V$ to $J$ then $D_{z}=\delta_{z}$. I.e.

$$
D_{z} f(s)=\left.\frac{d}{d t} f(s+t z)\right|_{t=0},
$$

is the (non-normalized) directional derivative in $V$.

(4) For $z, w \in V$ we have $D_{w}(z \mid s)=(z \mid \bar{w})$.

Suppose $z=x+i y, x, y \in J, x \neq 0, y \neq 0$. Then $z$ and $\bar{z}$ are independent over $\mathbb{C}$, and $\operatorname{span}_{\mathbb{C}}\{z, \bar{z}\}=\operatorname{span}_{\mathbb{C}}\{x, y\}$. On the other hand, if $z=x$ then $\operatorname{span}_{\mathbb{C}}\{z\}=\operatorname{span}_{\mathbb{C}}\{x\}$ and if $z=i y$ then $\operatorname{span}_{\mathbb{C}}\{z\}=\operatorname{span}_{\mathbb{C}}\{y\}$. It follows from these observations that there is an orthonormal basis of $V$ of the form

$$
\left\{e_{1}, \ldots, e_{n}, z_{1}, \overline{z_{1}}, \ldots, z_{k}, \overline{z_{k}}\right\},
$$

where $e_{1}, \ldots, e_{n} \in J$ and

$$
\left\{e_{1}, \ldots, e_{n}, x_{1}, \ldots, x_{k}, y_{1}, \ldots y_{k}\right\}
$$

where $z_{j}=x_{j}+i y_{j}, j=1, \cdots, k$, forms a basis of $\mathrm{J}$. We call such a basis of $V$ compatible with $J$.

Now let $J$ be the $\mathbb{R}$-vector space of $n \times n$ Hermitian symmetric spaces. I.e. $J=\left\{s \in M_{n, n}(\mathbb{C}): s=s^{*}\right\}$. On $V=M_{n, n}(\mathbb{C})$ there is a complex inner product given by $\left(z_{1} \mid z_{2}\right)=\operatorname{tr}\left(z_{1} z_{2}^{*}\right)$ which, when restricted to $J$, is a real scalar product. Notice also that conjugation with respect to the real form $\mathrm{J}$ is given by the adjoint *, i.e., $\bar{v}=v^{*}$. It is convenient to pick a specific basis for the calculations in the next section. Let $E_{i, j}$ be the $n \times n$ matrix with 1 in the $(i, j)$-entry and 0's elsewhere. Then the collection $\left\{E_{i, j}\right\}$ is a basis of $V$ compatible with $J$. For, observe that $E_{i, i}^{*}=E_{i, i}$ and $E_{i, j}^{*}=E_{j, i}, i \neq j$. Let $I_{i}=E_{i, i}, J_{i, j}=E_{i, j}+E_{j, i}$, and $K_{i, j}=$ $i E_{i, j}-i E_{j, i}$. (There should be no confusion between the index $i$ a positive integer and the complex number $i=\sqrt{-1}$. $)$ Then $E_{i, j}=\frac{1}{2}\left(J_{i, j}-i K_{i, j}\right)$. Furthermore

$$
\mathcal{B}=\left\{I_{i}, \frac{1}{2} J_{i, j}, \frac{1}{2} K_{i, j}\right\},
$$

where $i<j$, is a corresponding basis of $J$. Let $D_{i, j}=D_{E_{i, j}}=D_{\frac{1}{2}\left(J_{i, j}-i K_{i, j}\right)}$. Using this notation it follows that

$$
(\nabla)_{i, j} f:=(\nabla f)_{i, j}=\left(\nabla f \mid E_{i, j}\right)=D_{\bar{E}_{i, j}} f=D_{j, i} f .
$$

Furthermore, for $w \in V$,

$$
D_{w} f=\sum w_{i, j} D_{i, j} f=\sum w_{i, j}(\nabla)_{j, i} f=\operatorname{tr}(w \nabla) f,
$$

where $\operatorname{tr}$ is the usual trace functional.

\section{Proposition 3.2.}

(1) For $1 \leq i, j, k, l \leq n$ we have

$$
D_{i, j} s_{k, l}=\delta_{i, k} \delta_{j, l} .
$$

(2) Suppose $w \in V$ and $s \in J$ is invertible. Then

$$
D_{w} \operatorname{det}(s)^{m}=m \operatorname{det}(s)^{m} \operatorname{tr}\left(s^{-1} w\right) .
$$


(3) For $1 \leq i, j \leq n$ we have

$$
D_{i, j} \operatorname{det}(s)^{m}=m \operatorname{det}(s)^{m-1} \operatorname{cof}_{i, j}(s),
$$

where $\operatorname{cof}_{i, j}(s)$ denotes the $(i, j)$-cofactor of $s$

Proof. (1) is clear and (3) follows from (2). Since det is the restriction of a holomorphic function we have

$$
D_{w} \operatorname{det}(s)=\left.\frac{d}{d t} \operatorname{det}(s) \operatorname{det}\left(1+t s^{-1} w\right)\right|_{t=0}=\operatorname{det}(s) \operatorname{tr}\left(s^{-1} w\right) .
$$

Thus

$$
D_{w} \operatorname{det}(s)^{m}=m \operatorname{det}(s)^{m-1} D_{w} \operatorname{det}(s)=m \operatorname{det}(s)^{m} \operatorname{tr}\left(s^{-1} w\right) .
$$

Proposition 3.3. Suppose that $f, g \in L^{2}\left(\Omega, d \mu_{\nu}\right)$ are smooth and $f$ vanishes on the boundary of the cone $\Omega$. Let $1 \leq i, j \leq n$. Then

(1)

$$
\begin{aligned}
\int_{\Omega} D_{i, j} f(s) g(s) d s & =-\int_{\Omega} f(s) D_{i, j} g(s) d s . \\
\int_{\Omega} e^{-(z \mid s)} z_{i, j} f(s) d s & =\int_{\Omega} e^{-(z \mid s)} D_{j, i} f(s) d s .
\end{aligned}
$$

Proof. (1) is Stokes Theorem and (2) follows from (1) and the fact that $D_{j, i} e^{-(z \mid s)}=$ $-e^{-(z \mid s)} z_{i, j}$

\section{THE ACTION OF $\mathfrak{g}_{\mathbb{C}}$ ON $L^{2}\left(\Omega, d \mu_{\nu}\right)$}

In section 2 we determined the algebraic action of $\mathfrak{g}_{\mathbb{C}}$ on $\mathcal{H}_{\nu}(T(\Omega))$. Recall the measure $d \mu_{\nu}(t)=\operatorname{det}(t)^{\nu-n} d t$ and the inner product $(x \mid y)=\operatorname{tr}(x y)$. The Laplace transform

$$
\mathcal{L}_{\nu}: L^{2}\left(\Omega, d \mu_{\nu}\right) \rightarrow \mathcal{H}_{\nu}(T(\Omega))
$$

given by the formula

$$
\mathcal{L}_{\nu}(f)(z)=\int_{\Omega} e^{-(z \mid s)} f(s) \operatorname{det}(s)^{\nu-n} d s=\int_{\Omega} e^{-(z \mid s)} f(s) d \mu_{\nu}(s),
$$

defines an isomorphism between $L^{2}\left(\Omega, d \mu_{\nu}\right)$ and $\mathcal{H}_{\nu}(T(\Omega))$. We can thus define an equivalent action, denoted $\lambda_{\nu}$, of $\mathfrak{g}_{\mathbb{C}}$ on $L^{2}\left(\Omega, d \mu_{\nu}\right)$. This action is given in the following theorem.

Theorem 4.1. Let $f \in L^{2}\left(\Omega, d \mu_{\nu}\right)$ be a smooth vector.

(1) Let $\left(\begin{array}{ll}a & b \\ b & a\end{array}\right) \in \mathfrak{k}_{\mathbb{C}}$. Then

$$
\lambda_{\nu}\left(\begin{array}{ll}
a & b \\
b & a
\end{array}\right) f(s)=\operatorname{tr}(-s \nabla b \nabla+(s a-a s-\nu b) \nabla+b s) f(s)
$$

(2) Let $\left(\begin{array}{cc}x & x \\ -x & -x\end{array}\right) \in \mathfrak{p}^{+}$. Then

$$
\lambda_{\nu}\left(\begin{array}{cc}
x & x \\
-x & -x
\end{array}\right) f(s)=\operatorname{tr}(s \nabla x \nabla+(\nu x+s x+x s) \nabla+(\nu x+s x)) f(s) .
$$


(3) Let $\left(\begin{array}{ll}x & -x \\ x & -x\end{array}\right) \in \mathfrak{p}^{-}$. Then

$$
\lambda_{\nu}\left(\begin{array}{ll}
x & -x \\
x & -x
\end{array}\right) f(s)=\operatorname{tr}(-s \nabla x \nabla+(-\nu x+s x+x s) \nabla+(\nu x-s x)) f(s)
$$

The proof of this theorem is somewhat long and certainly tedious. We will provide the details for the subalgebra $\mathfrak{p}^{+}$. The actions for the other algebras are done in a similar manner. Throughout we will use without reference the results of section 3 .

4.1. (2) The proof for the $\mathfrak{p}^{+}$- action. Let $X=\left(\begin{array}{cc}x & x \\ -x & -x\end{array}\right) \in \mathfrak{p}^{+}$and let $f \in L^{2}\left(\Omega, d \mu_{\nu}\right)$ be a smooth vector. We use the Laplace transform $\mathcal{L}_{\nu}$ to transfer the action of $\mathfrak{p}^{+}$on $L^{2}\left(\Omega, d \mu_{\nu}\right)$. Thus we seek a formula for $\lambda_{\nu}(X)$ such that

$$
\pi_{\nu}(X) \mathcal{L}_{\nu} f(z)=\mathcal{L}_{\nu}\left(\lambda_{\nu}(X) f\right)(z)
$$

Recall from Theorem 2.1 that for $F$ a smooth vector in $\mathcal{H}_{\nu}(T(\Omega))$ we have

$$
\pi_{\nu}(X) F(z)=-\nu \operatorname{tr}(x(1+z)) F(z)-\delta((1+z) x(1+z)) F(z) .
$$

Our first calculation will begin with $-\delta((z+1) x(z+1)) \mathcal{L}_{\nu} f(z)$. Let $m=\nu-d / r=$ $\nu-n$.

\section{Lemma 4.2.}

$$
\begin{aligned}
-\delta((z+1) x(z+1)) \mathcal{L}_{\nu} f(z)= & -\delta((z+1) x(z+1)) \int e^{-(z \mid s)} f(s) \operatorname{det}(s)^{m} d s \\
= & \int e^{-(z \mid s)}((z+1) x(z+1) \mid s) f(s) \operatorname{det}(s)^{m} d s \\
= & \int e^{-(z \mid s)}(x \mid s) f(s) \operatorname{det}(s)^{m} d s \\
& +\int e^{-(z \mid s)}(x z \mid s) f(s) \operatorname{det}(s)^{m} d s \\
& +\int e^{-(z \mid s)}(z x \mid s) f(s) \operatorname{det}(s)^{m} d s \\
& +\int e^{-(z \mid s)}(z x z \mid s) f(s) \operatorname{det}(s)^{m} d s .
\end{aligned}
$$

Lemma $4.3(\mathrm{~B})$.

$$
\int e^{-(z \mid s)}(x z \mid s) f(s) \operatorname{det}(s)^{m} d s=(n+m) \operatorname{tr}(x) \mathcal{L}_{\nu} f(z)+\int e^{-(z \mid s)} \operatorname{tr}(s x \nabla) f(s) \operatorname{det}(s)^{m} d s .
$$


Proof. First observe that $(x z \mid s)=\sum_{i, j}(x z)_{i, j} s_{i, j}^{*}=\sum_{i, j, k} x_{i, k} z_{k, j} s_{j, i}$.

$$
\begin{aligned}
B= & \sum_{i, k, j} \int e^{-(z \mid s)} x_{i, k} D_{j, k}\left(s_{j, i} f(s) \operatorname{det}(s)^{m}\right) d s \\
= & \sum_{i, k, j} \int e^{-(z \mid s)} x_{i, k} \delta_{k, i} f(s) \operatorname{det}(s)^{m} d s \\
& \quad+\sum_{i, k, j} \int e^{-(z \mid s)} x_{i, k} s_{j, i}\left(D_{j, k} f\right)(s) \operatorname{det}(s)^{m} d s \\
& +\sum_{i, k, j} \int e^{-(z \mid s)} x_{i, k} s_{j, i} f(s) m \operatorname{det}(s)^{m-1} \operatorname{cof}_{j, k}(s) d s \\
= & (n+m) \operatorname{tr}(x) \mathcal{L}_{\nu} f(z)+\sum_{j, k} \int e^{-(z \mid s)}(s x)_{j, k}\left(D_{j, k} f\right)(s) \operatorname{det}(s)^{m} d s \\
= & (n+m) \operatorname{tr}(x) \mathcal{L}_{\nu} f(z)+\int e^{-(z \mid s)} \operatorname{tr}(s x \nabla) f(s) \operatorname{det}(s)^{m} d s .
\end{aligned}
$$

\section{Lemma $4.4(\mathrm{C})$.}

$$
\int e^{-(z \mid s)}(z x \mid s) f(s) \operatorname{det}(s)^{m} d s=(n+m) \operatorname{tr}(x) \mathcal{L}_{\nu} f(z)+\int e^{-(z \mid s)} \operatorname{tr}(x s \nabla) f(s) \operatorname{det}(s)^{m} d s .
$$

Proof. This proof is very similar to the proof of lemma (4.3). It is omitted.

Putting $\mathrm{B}$ and $\mathrm{C}$ together gives

\section{Lemma 4.5.}

$$
B+C=2(n+m) \operatorname{tr}(x) \mathcal{L}_{\nu} f(z)+\mathcal{L}_{\nu}(\operatorname{tr}((x s+s x) \nabla) f)(z) .
$$

We now calculate D and obtain

Lemma 4.6 (D).

$$
\begin{aligned}
D= & (n+m) \operatorname{tr}(z x) \mathcal{L}_{\nu} f(z) \\
& +(n+m) \mathcal{L}_{\nu}(\operatorname{tr}(x \nabla) f)(z) \\
& +\mathcal{L}_{\nu}(\operatorname{tr}(s \nabla x \nabla) f)(z)
\end{aligned}
$$

Proof. First observe that

$$
(z x z \mid s)=\sum_{i, j, k, l} z_{i, k} x_{k, l} z_{l, j} s_{j, i}
$$


Thus

$$
\begin{aligned}
& D=\sum_{i, k, j, l} \int e^{-(z \mid s)} z_{i, k} z_{l, j} s_{j, i} x_{k, l} f(s) \operatorname{det}(s)^{m} d s \\
& =\sum_{i, k, j, l} x_{k, l} z_{i, k} \int e^{-(z \mid s)} D_{j, l}\left(s_{j, i} f(s) \operatorname{det}(s)^{m}\right) d s \\
& =\sum_{i, k, j, l} x_{k, l} z_{i, k} \int e^{-(z \mid s)} \delta_{l, i} f(s) \operatorname{det}(s)^{m} d s \\
& +\sum_{i, k, j, l} x_{k, l} z_{i, k} \int e^{-(z \mid s)} s_{j, i}\left(D_{j, l} f\right)(s) \operatorname{det}(s)^{m} d s \\
& +\sum_{i, k, j, l} x_{k, l} z_{i, k} \int e^{-(z \mid s)} s_{j, i} f(s) m \operatorname{det}(s)^{m-1} \operatorname{cof}_{j, l}(s) d s \\
& =(n+m) \sum_{k, l} x_{k, l} z_{l, k} \int e^{-(z \mid s)} f(s) \operatorname{det}(s)^{m} d s \\
& +\sum_{i, k, j, l} x_{k, l} z_{i, k} \int e^{-(z \mid s)} s_{j, i}\left(D_{j, l} f\right)(s) \operatorname{det}(s)^{m} d s \\
& =(n+m) \operatorname{tr}(z x) \mathcal{L}_{\nu} f(z)+\sum_{i, k, j, l} x_{k, l} \int e^{-(z \mid s)} D_{k, i}\left(s_{j, i}\left(D_{j, l} f\right)(s) \operatorname{det}(s)^{m}\right) d s \\
& =(n+m) \operatorname{tr}(z x) \mathcal{L}_{\nu} f(z) \\
& +\sum_{i, k, j, l} x_{k, l} \int e^{-(z \mid s)} \delta_{k, j}\left(D_{j, l} f\right)(s) \operatorname{det}(s)^{m} d s \\
& +\sum_{i, k, j, l} x_{k, l} \int e^{-(z \mid s)} s_{j, i}\left(D_{k, i} D_{j, l} f\right)(s) \operatorname{det}(s)^{m} d s \\
& +\sum_{i, k, j, l} x_{k, l} \int e^{-(z \mid s)} s_{j, i}\left(D_{j, l} f\right)(s) m \operatorname{det}(s)^{m-1} \operatorname{cof}_{k, i}(s) d s \\
& =(n+m) \operatorname{tr}(z x) \mathcal{L}_{\nu} f(z) \\
& +(n+m) \int e^{-(z \mid s)} \sum_{k, l} x_{k, l}\left(D_{k, l} f\right)(s) \operatorname{det}(s)^{m} d s \\
& +\int e^{-(z \mid s)} \sum_{i, k, j, l} s_{j, i}\left(D_{k, i} x_{k, l}\left(D_{j, l} f\right)\right)(s) \operatorname{det}(s)^{m} d s \\
& =(n+m) \operatorname{tr}(z x) \mathcal{L}_{\nu} f(z) \\
& +(n+m) \mathcal{L}_{\nu}(\operatorname{tr}(x \nabla) f)(z) \\
& +\mathcal{L}_{\nu}(\operatorname{tr}(s \nabla x \nabla) f)(z) \text {. }
\end{aligned}
$$

By putting together lemmas (4.2)-(4.6) we obtain 


\section{Lemma 4.7.}

$$
\begin{aligned}
-\delta((z+1) x(z+1)) \mathcal{L}_{\nu} f(z)= & \mathcal{L}_{\nu}(\operatorname{tr}(s x) f)(z)+2(n+m) \operatorname{tr}(x) \mathcal{L}_{\nu} f(z) \\
& +\mathcal{L}_{\nu}\left(D_{x s+s x} f\right)(z)+(n+m) \operatorname{tr}(z x) \mathcal{L}_{\nu}(f)(z) \\
& +(n+m) \mathcal{L}_{\nu}(\operatorname{tr}(x \nabla) f)(z)+\mathcal{L}_{\nu}(\operatorname{tr}(s \nabla x \nabla) f)(z) .
\end{aligned}
$$

We thus obtain

$$
\begin{aligned}
\pi_{\nu}\left(\left(\begin{array}{cc}
x & x \\
-x & -x
\end{array}\right) \mathcal{L}_{\nu}(f)(z)=\right. & -\nu \operatorname{tr}(x(z+1)) \mathcal{L}_{\nu} f(z)-\delta\left((z+1) x(z+1) \mathcal{L}_{\nu} f(z)\right. \\
= & -\nu \operatorname{tr}(x z) \mathcal{L}_{\nu} f(z)-\nu \operatorname{tr}(x) \mathcal{L}_{\nu} f(z) \\
& +\mathcal{L}_{\nu}(\operatorname{tr}(s x) f)(z)+2 \nu \operatorname{tr}(x) \mathcal{L}_{\nu} f(z) \\
& +\mathcal{L}_{\nu}(\operatorname{tr}((s x+x s) \nabla) f)(z)+\nu \operatorname{tr}(z x) \mathcal{L}_{\nu} f(z) \\
& +\nu \mathcal{L}_{\nu}(\operatorname{tr}(x \nabla) f)(z)+\mathcal{L}_{\nu}(\operatorname{tr}(s \nabla x \nabla) f)(z) \\
= & \nu \operatorname{tr}(x) \mathcal{L}_{\nu} f(z)+\mathcal{L}_{\nu}(\operatorname{tr}(s x) f)(z) \\
& +\mathcal{L}_{\nu}(\operatorname{tr}((s x+x s) \nabla) f)(z) \\
& +\nu \mathcal{L}_{\nu}(\operatorname{tr}(x \nabla) f)(z)+\mathcal{L}_{\nu}(\operatorname{tr}(s \nabla x \nabla) f)(z) \\
= & \mathcal{L}_{\nu}(\operatorname{tr}((\nu x+s x)+(\nu x+s x+x s) \nabla+s \nabla x \nabla) f)(z)
\end{aligned}
$$

This completes the proof.

\section{Recursion Relations}

We will now state the main recursion relations implied by these actions. The notation and definition for the Laguerre functions are in [2], section 7.

Theorem 5.1. The Laguerre functions are related by the following recursion relations:

(1) $\operatorname{tr}(-s \nabla \nabla-\nu \nabla+s) \ell_{\mathbf{m}}^{\nu}=(r \nu+2|\mathbf{m}|) \ell_{\mathbf{m}}^{\nu}$

(2) $\frac{1}{2} \operatorname{tr}(s \nabla \nabla+(\nu I+2 s) \nabla+(\nu I+s)) \ell_{\mathbf{m}}^{\nu}(s)=-\sum_{j=1}^{r}\left(\begin{array}{c}\mathbf{m} \\ \mathbf{m}-\gamma_{\mathbf{j}}\end{array}\right)\left(m_{j}-1+\nu-\right.$ $(j-1)) \ell_{\mathbf{m}-\gamma_{\mathrm{j}}}^{\nu}$

(3) $\frac{1}{2} \operatorname{tr}(-s \nabla \nabla+(-\nu I+2 s) \nabla+(\nu I-s)) \ell_{\mathbf{m}}^{\nu}=\sum_{j=1}^{r} c_{\mathbf{m}}(j) \ell_{\mathbf{m}+\gamma_{\mathbf{j}}}^{\nu}$.

Proof. Let $\xi=\left(\begin{array}{ll}0 & 1 \\ 1 & 0\end{array}\right)$. Then by lemma 5.5 of $[2]$

$$
\pi_{\nu}(\xi) q_{\mathbf{m}, \nu}=(r \nu+2|\mathbf{m}|) q_{\mathbf{m}, \nu},
$$

where, by theorem 7.8 of [2], $\mathcal{L}_{\nu}\left(\ell_{\mathbf{m}}^{\nu}\right)=\Gamma_{\Omega}(\mathbf{m}+\nu) q_{\mathbf{m}, \nu}$. From this it follows that

$$
\begin{aligned}
\lambda_{\nu}(\xi) \ell_{\mathbf{m}}^{\nu} & =\mathcal{L}_{\nu}^{-1} \pi_{\nu}(\xi) \mathcal{L}_{\nu} \ell_{\mathbf{m}}^{\nu} \\
& =\mathcal{L}_{\nu}^{-1} \pi_{\nu}(\xi) \Gamma_{\nu}(\mathbf{m}+\nu) q_{\mathbf{m}, \nu} \\
& =(r \nu+2|\mathbf{m}|) \mathcal{L}_{\nu}^{-1}\left(\Gamma_{\Omega}(\mathbf{m}+\nu) q_{\mathbf{m}, \nu}\right) \\
& =(r \nu+2|\mathbf{m}|) \ell_{\mathbf{m}}^{\nu} .
\end{aligned}
$$

On the other hand, if $a=0$ and $b=1$ in Theorem 4.1 then

$$
\lambda_{\nu}(\xi) \ell_{\mathbf{m}}^{\nu}=\operatorname{tr}(-s \nabla \nabla-\nu \nabla+s) \ell_{\mathbf{m}}^{\nu}
$$

and part (1) follows. 
The vector $\xi$ is in the center of $\mathfrak{k}_{\mathbb{C}}$ and $\operatorname{ad}(\xi)$ acts by $-2 I$ on $\mathfrak{p}^{+}$and $2 I$ on $\mathfrak{p}^{-}$, where $I$ is the identity operator. Let

$$
L_{k}^{2}\left(\Omega, d \mu_{\nu}\right)=\left\{f \in L^{2}\left(\Omega, d \mu_{\nu}\right): \lambda_{\nu}(\xi) f=(r \nu+2 k) f\right\} .
$$

Since $\lambda_{\nu}$ is an irreducible highest weight representation it is well known that $L_{k}^{2}\left(\Omega, d \mu_{\nu}\right)$ is finite dimensional, nonzero if $k \geq 0$, and $L^{2}\left(\Omega, d \mu_{\nu}\right)=\oplus L_{k}^{2}\left(\Omega, d \mu_{\nu}\right)$. Furthermore, part (1) implies that $\ell_{\mathbf{m}}^{\nu} \in L_{|\mathbf{m}|}^{2}\left(\Omega, d \mu_{\nu}\right)$, for all $\mathbf{m} \in \Lambda$ (c.f. (2.15) of [2]). For $x \in \mathfrak{p}^{+}$and $f \in L_{k}^{2}\left(\Omega, d \mu_{\nu}\right)$ we have

$$
\begin{aligned}
\lambda_{\nu}(\xi) \lambda_{\nu}(x) f & =\lambda_{\nu}(x) \lambda_{\nu}(\xi) f+\lambda_{\nu}([\xi, x]) f \\
& =(r \nu+2 k) \lambda_{\nu}(x) f-2 \lambda_{\nu}(x) f \\
& =(r \nu+2(k-1)) \lambda_{\nu}(x) f .
\end{aligned}
$$

This implies that $\lambda_{\nu}(x) f \in L_{k-1}^{2}\left(\Omega, d \mu_{\nu}\right)$. Similarly, for $x \in \mathfrak{p}^{-}$, we have $\lambda_{\nu}(x) f \in$ $L_{k+1}^{2}\left(\Omega, d \mu_{\nu}\right)$.

Now let $X^{+}=\left(\begin{array}{cc}-1 & -1 \\ 1 & 1\end{array}\right) \in \mathfrak{p}^{+}, X^{-}=\left(\begin{array}{cc}1 & -1 \\ 1 & -1\end{array}\right) \in \mathfrak{p}^{-}$, and $Z=\frac{1}{2}\left(X^{-}-\right.$ $\left.X^{+}\right)=\left(\begin{array}{cc}1 & 0 \\ 0 & -1\end{array}\right)$. Then by theorem 7.9 of [2] and its proof

$$
\lambda_{\nu}(Z) \ell_{\mathbf{m}}^{\nu}=-\sum_{j=1}^{r}\left(\begin{array}{c}
m \\
m-\gamma_{j}
\end{array}\right)\left(m_{j}-1+\nu-(j-1)\right) \ell_{m-\gamma_{j}}^{\nu}+\sum_{j=1}^{r} c_{m}(j) \ell_{m+\gamma_{j}}^{\nu} .
$$

If $P_{k}$ denotes the orthogonal projection of $L^{2}\left(\Omega, d \mu_{\nu}\right)$ onto $L_{k}^{2}\left(\Omega, d \mu_{\nu}\right)$ then

$$
\frac{-1}{2} \lambda_{\nu}\left(X^{+}\right) \ell_{\mathbf{m}}^{\nu}=P_{|\mathbf{m}|-1} \lambda_{\nu}(Z) \ell_{\mathbf{m}}^{\nu}=-\sum_{j=1}^{r}\left(\begin{array}{c}
m \\
m-\gamma_{j}
\end{array}\right)\left(m_{j}-1+\nu-(j-1)\right) \ell_{m-\gamma_{j}}^{\nu}
$$

and

$$
\frac{1}{2} \lambda_{\nu}\left(X^{-}\right) \ell_{\mathbf{m}}^{\nu}=P_{|\mathbf{m}|+1} \lambda_{\nu}(Z) \ell_{\mathbf{m}}^{\nu}=\sum_{j=1}^{r} c_{m}(j) \ell_{m+\gamma_{j}}^{\nu}
$$

Formulas (2) and (3) now follow from theorem 4.1 part (2) and (3), respectively, by setting $x=1$.

\section{REFERENCES}

[1] M. Davidson, G. Ólafsson, and Genkai Zhang, Laguerre Polynomials, Restriction Principle, and Holomorphic Representations of $\mathrm{SL}(2, \mathbb{R})$ Acta Applicandae Mathematicae $\mathbf{7 1}$ (2002), 261-277.

[2] M. Davidson, G. Ólafsson, and Genkai Zhang, Segal-Bargmann Transform on Hermitian Symmetric Spaces and Orthogonal Polynomials. Submitted. http://arxiv.org/math.RT/0206275

[3] J. Faraut and A. Koranyi, Function spaces and reproducing kernels on bounded symmetric domains, J. Funct. Anal. 88 (1990), 64-89.

[4] S. G. Gindikin, Analysis on homogeneous domains, Uspekhi Mat. Nauk, 19, No 4, 3-92; Russian Math. Surveys, 19, No. 4, 1-89.

[5] S. Helgason,Differential Geometry, Lie Groups, and Symmetric Spaces, Academic Press, 1978.

[6] B. Kostant, On Laguerre Polynomials, Bessel Functions, Hankel Transform and a series in the Unitary Dual of the Simply-Connected Covering group of $\mathrm{SL}(2, \mathbb{R})$. Representation Theory, 4 (2000), 181-224. 
[7] G. Ólafsson and B. Ørsted, Generalizations of the Bargmann transform, Lie theory and its applications in physics. Proceedings of the international workshop, Clausthal, Gemany, August 14-17, 1995. (H.-D. Doebner et al, ed.), World scientific, Singapore, 1996, pp. 3-14, MR. 99c:22017.

[8] F. Ricci and A. Vignati, Bergman spaces on some tube-type domains and Laguerre operators on symmetric cones, J. Reine Angew. Math., 449 (1994), 81-101.

[9] H. Rossi and M. Vergne, Analytic continuation of the holomorphic discrete series of a semisimple Lie group, Acta Math. 136 (1976), 1-59.

[10] N. Wallach, The analytic continuation of the discrete series, I, II Trans. Amer. Math. Soc. 251 (1979), 1-17; 19-37.

[11] N. Wallach, Generalized Whittaker Vectors for Holomorphic and Quaternionic Representations. Preprint.

Department of Mathematics, Louisiana State University, Baton Rouge, LA 70803, USA

E-mail address: davidson@math.1su.edu

E-mail address: olafsson@math.1su.edu 